\title{
Hypoxia-induced Preadipocyte factor 1 expression in human lung fibroblasts through ERK/PEA3/c-Jun pathway
}

\section{Wun-Hao Cheng}

Taipei Medical University

Chia-Ling Chen

Taipei Medical University

Jing-Yun Chen

Taipei Medical University

Chien-Huang Lin

Taipei Medical University

Bing-Chang Chen ( $\boldsymbol{\sigma}$ bcchen@tmu.edu.tw )

Taipei Medical University https://orcid.org/0000-0003-1926-9643

\section{Research Article}

Keywords: hypoxia, Pref-1, ERK, PEA3, AP-1, human lung fibroblasts

Posted Date: April 12th, 2021

DOI: https://doi.org/10.21203/rs.3.rs-400752/v1

License: (c) (1) This work is licensed under a Creative Commons Attribution 4.0 International License.

Read Full License

Version of Record: A version of this preprint was published at Molecular Medicine on July 6th, 2021. See the published version at https://doi.org/10.1186/s10020-021-00336-w. 


\section{Abstract \\ Background}

Several studies have reported that hypoxia plays a pathological role in severe asthma and tissue fibrosis. Our previous study showed that hypoxia induces A disintegrin and metalloproteinase 17 (ADAM17) expression in human lung fibroblasts. Moreover, preadipocyte factor 1 (Pref-1) is cleaved by ADAM17, which participates in adipocyte differentiation. Furthermore, Pref1 overexpression is involved in liver and heart fibrosis. Studies have demonstrated that polyoma enhancer activator 3 (PEA3) and activator protein 1 (AP-1) play crucial roles in lung fibrosis, and the Pref-1 promoter region contains PEA3 and AP-1 binding sites, as predicted. However, the roles of extracellular signal-regulated kinase (ERK), PEA3, and AP-1 in hypoxia-stimulated Pref-1 expression in human lung fibroblasts remain unknown.

\section{Methods}

The protein expression in ovalbumin (OVA)-induced asthmatic mice was performed by immunohistochemistry and immunofluorescence. The protein expression or the mRNA level in human lung fibroblasts (WI-38) was detected by western blot or quantitative PCR. Small interfering (si) RNA was used to knockdown gene expression. The collaboration with PEA3 and c-Jun were determined by coimmunoprecipitation. Translocation of PEA3 from the cytosol to the nucleus was observed by immunocytochemistry. The binding ability of PEA3 and AP-1 to Pref-1 promoter was assessed by chromatin immunoprecipitation.

\section{Results}

Pref-1, PEA3, and hypoxia-inducible factor $1 \mathrm{a}(\mathrm{HIF-1}$ a) were expressed in the lung sections of ovalbumintreated mice. Hypoxia induced Pref1 protein upregulation and mRNA expression in human lung fibroblasts (WI38 cells). Moreover, PEA3 small interfering (si) RNA decreased the expression of hypoxiainduced Pref1 in WI38 cells. Hypoxia induced PEA3 phosphorylation, translocation of PEA3 from the cytosol to the nucleus, PEA3 recruitment and AP-1 binding to the Pref1 promoter region, and PEA3luciferase activity. Furthermore, hypoxia induced c-Jun-PEA3 complex formation, and U0126 (an ERK inhibitor) or curcumin (an AP1 inhibitor) downregulated hypoxia-induced Pref-1 expression.

\section{Conclusions}

These results implied that ERK, PEA3, and AP1 participate in hypoxiainduced Pref1 expression in human lung fibroblasts.

\section{Introduction}


Asthma is an allergic airway disease characterized by airway inflammation, epithelial apoptosis, and airway remodeling $(1,2)$. A study showed that approximately $3.7 \%$ of patients with asthma developed severe asthma (3). Severe asthma is a difficult-to-control airway disease; it requires inhalation of high doses of corticosteroids to relieve symptoms (4). Airway fibrosis occurs when fibroblasts differentiate into a-smooth muscle actin myofibroblasts with massive deposition of extracellular matrix, including collagen I (5-7). Numerous studies have shown that subepithelial collagen deposition and fibrosis are correlated with asthma severity $(5,8,9)$.

Hypoxia contributes to the fibrosis of several organs, including the kidney, liver, and lung (10-13). A study indicated that hypoxia plays a vital role in asthma (1). Hypoxia regulates gene expression through hypoxia-inducible factors (HIFs), and HIF-a is a key transcript factor produced in response to hypoxia (14). Airway inflammation and fibroblast proliferation cause airway remodeling, leading to hypoxia $(1,15$, 16). Furthermore, hypoxia exacerbates airway remodeling and fibrosis (17-19). Thus, hypoxia plays a major role in airway fibrosis.

Preadipocyte factor 1 (Pref-1) is a transmembrane protein and is processed to the soluble protein by $A$ disintegrin and metalloproteinase 17 (ADAM17; 20 ). Pref-1 was originally found to be responsible for adipogenesis inhibition in preadipocytes (21). A study reported that Pref- 1 is involved in mesenchymal cell differentiation (22). Moreover, soluble Pref- 1 participates in heart and liver fibrosis $(23,24)$. However, the role of Pref- 1 in human lung fibrosis remains unknown. A study indicated that hypoxia induces ADAM17 expression in human lung fibroblasts, and ADAM17 is involved in hypoxia-induced fibroblast differentiation (25). Whether hypoxia can induce Pref-1 expression in human lung fibroblasts needs further investigation.

Polyoma enhancer activator 3 (PEA3), also called ETs translocation variant 4, is a transcription factor and plays a crucial role in cancer cell metastasis and fibrotic gene expression $(26,27)$. Studies have revealed that extracellular signal-regulated kinase (ERK)-induced PEA3 phosphorylation mediate gene expression in collaboration with another transcription factor $(28,29)$. According to the NCBI database, the PEA3 binding sequence is present in the Pref-1 promoter. However, the role of PEA3 in hypoxia-induced Pref-1 needs clarification.

The activator protein 1 (AP-1) transcript factor is a heterodimer composed of c-Jun and c-Fos. It usually regulates gene expression in cell growth, differentiation, and apoptosis (30). A study showed that AP-1 is involved in profibrotic protein expression in lung fibrosis (31). Moreover, hypoxia activates AP-1, which induces Cyr61 expression in retinal vascular endothelial cells (30). In hepatoma cells, PEA3 regulates IL8/CXCL8 gene expression in collaboration with AP-1 (32). Nevertheless, whether hypoxia induces Pref-1 expression through the ERK/PEA3/AP-1 pathway remains unknown.

In the present study, we revealed that hypoxia increased ERK phosphorylation, which in turn induced PEA3 and AP-1 activation and theirs recruitment to the Pref-1 promoter region to increase Pref-1 expression in human lung fibroblasts. Examining the mechanism may provide a potential maker of airway fibrosis in patients with severe asthma. 


\section{Materials And Methods \\ 2.1 Materials}

Antibodies specific for Pref-1, HIF-1a, and phospho-serine were purchased from Abcam (Cambridge, MA, USA). Secondary antibodies against IP detection reagent, Alexa Fluor-488 and Alexa Fluor-555 were purchased from Abcam (Cambridge, MA, USA). An antibody specific for c-Jun Ser63 was purchased from Cell Signaling Technology (Danvers, MA, USA). Antibodies specific for PEA3, c-Jun, horseradish peroxidase (HRP)-linked antibodies, including anti-goat immunoglobulin $\mathrm{G}(\mathrm{IgG})$, anti-rabbit lgG, and antimouse IgG antibodies, were obtained from Santa Cruz Biotechnology (Santa Cruz, CA, USA). A luciferase assay kit was purchased from Promega (Madison, WI, USA). The human PEA3 luciferase reporter was a obtained from Peter Hollenhorst (Watertown, MA, USA). Furthermore, a-tubulin antibody, fetal bovine serum, control small interfering RNA (siRNA) (scrambled), and PEA3 siRNA were purchased from SigmaAldrich (St. Louis, MO, USA). Lipofectamine 3000 reagent, minimum essential medium (MEM), penicillin, and streptomycin were acquired from Invitrogen Life Technologies (Carlsbad, CA, USA). A Novolink Max Polymer Detection System was purchased from Leica (Wetzlar, Germany)

\subsection{Cell culture}

Human lung fibroblast (WI-38) cells, purchased from American Type Culture Collection (Manassas, VA, USA), were grown in MEM supplemented with $10 \%$ fetal calf serum, penicillin $\mathrm{G}(100 \mathrm{U} / \mathrm{mL})$, streptomycin $(100 \mu \mathrm{g} / \mathrm{mL})$, and MEM nonessential amino acids. WI-38 cells were maintained in a humidified $37^{\circ} \mathrm{C}$ incubator with $5 \% \mathrm{CO}_{2}$. After reaching confluence, cells were seeded onto 12-well plates for the transfection and luciferase reporter assay, onto 6-cm dishes for Western blot analysis, and onto 10-cm dishes for the immunoprecipitation assay.

\subsection{Ovalbumin-induced animal model of airway fibrosis}

For sensitization, on days 1, 7, and 14, C57BL/ 6 mice were intraperitoneally injected with $200 \mu \mathrm{L}$ of $50 \mu \mathrm{g}$ ovalbumin (OVA) emulsified in $2 \mathrm{mg}$ of aluminum hydroxide. From day 21, 8- to 10-week-old C57BL/6 mice were challenged with aerosolized 5\% OVA in phosphate-buffered saline (PBS) or PBS alone for 9 weeks. The frequency of the OVA challenge was twice weekly. After the final OVA aerosol challenge, C57BL/ 6 mice were sacrificed, and the lung tissue of mice was analyzed with further experiments.

\subsection{Western blot analysis}

Protein extraction and Western blot analysis were performed as previously described (33). In brief, cells were lysed with lysis buffer containing $20 \mathrm{mM}$ Tris (pH 7.5), $1 \mathrm{mM} \mathrm{MgCl}, 125 \mathrm{mM} \mathrm{NaCl}_{2}, 1 \%$ Triton X100, $1 \mathrm{mM}$ PMSF, $10 \mu \mathrm{g} / \mathrm{mL}$ leupeptin, $10 \mu \mathrm{g} / \mathrm{mL}$ aprotinin, $25 \mathrm{mM} \beta$-glycerophosphate, $50 \mathrm{mM} \mathrm{NaF}$, and $100 \mu \mathrm{M} \mathrm{Na}_{3} \mathrm{VO}_{4}$. Whole cell lysates $(30 \mu \mathrm{g})$ were electrophoresed through SDS-PAGE, and the gels were transferred onto PVDF membranes. The whole membranes were blocked through incubation with $5 \%$ bovine serum albumin for 1 hour. Subsequently, proteins were incubated with specific primary antibodies for 20 hours at $4^{\circ} \mathrm{C}$. Then, the membranes were incubated with HRP-conjugated secondary antibody for 1 
hour at room temperature. Immunoreactivity was analyzed using enhanced chemiluminescence following the manufacturer's protocol.

\subsection{Cell transfection}

For transient transfection of siRNA into WI-38 cells, the transfection reagent/siRNA mixture was incubated at room temperature for 10 minutes and then added dropwise to MEM supplemented with $10 \%$ fetal bovine serum; it was then incubated in a humidified $37^{\circ} \mathrm{C}$ incubator for 24 hours.

\subsection{PEA3-luciferase activity assay}

In brief, WI-38 cells were transfected with PEA3-Luc $(0.8 \mu \mathrm{g})$ and pBK-CMV-Lac Z $(0.1 \mu \mathrm{g})$ by using transfection reagent for 24 hours. Cells were stimulated with hypoxia $\left(1 \% \mathrm{O}_{2}\right)$ for 18 hours. Luciferase activity was measured using the luciferase assay kit (Promega, Madison, WI, USA).

\subsection{Immunohistochemistry and immunofluorescence}

Lung tissues were fixed in 10\% formaldehyde overnight, embedded in paraffin, and sectioned for immunohistochemistry (IHC) and immunofluorescence staining. For IHC staining, 2- $\mu$ m sections were deparaffinized and then processed for antigen retrieval by using citrate buffer $(\mathrm{pH} 6.0)$. Endogenous peroxidase in tissue was neutralized through peroxidase blocking. The tissue was then blocked with a blocking buffer and incubated with Pref- 1 and PEA3 antibodies for 40 minutes followed by polymer secondary antibody incubation for 30 minutes. Finally, the tissues were stained with hematoxylin and DAB solution. The integrated density of IHC staining was analyzed using ImageJ Fiji software. For immunofluorescence staining, $2-\mu \mathrm{m}$ sections were deparaffinized and then processed for antigen retrieval by using EDTA buffer ( $\mathrm{pH}$ 9.0). The tissue was blocked with a blocking buffer and incubated with Pref-1 and HIF-1a antibody for 24 hours at $4^{\circ} \mathrm{C}$. Then, the tissue was incubated with Alexa Fluor 488-conjugated and Alexa Fluor 555-conjugated secondary antibodies for 1 hour at room temperature. Images were scanned using a ScanScope CS or fluorescence microscope.

\subsection{Immunocytochemistry}

WI-38 cells were cultured on slides. After reaching confluence, cells were subjected to hypoxia ( $\left.1 \% \mathrm{O}_{2}\right)$ for 30 minutes and then fixed through incubation in 4\% paraformaldehyde in PBS for 10 minutes at room temperature. Permeabilization with PBS containing either $0.5 \%$ Triton X-100, and washed slides in PBS three times for 5 minutes. The coverslips were blocked with 5\% bovine serum albumin in PBST for 1 hour and incubated at $4^{\circ} \mathrm{C}$ overnight with antibodies specific to PEA3. Cells were incubated with Alexa Fluor 488-conjugated secondary antibody for an additional 1 hour. Counter staining was performed DAPI for 1 minute and the coverslip was mounted with a mounting medium. They were then observed under a fluorescence microscope.

\subsection{Coimmunoprecipitation}

WI-38 cells were seeded onto 10-cm dishes. After reaching confluence, cells were subjected to hypoxia $\left(1 \% \mathrm{O}_{2}\right)$ for the indicated time intervals. Cells were then harvested, lysed in $100 \mu \mathrm{L}$ of IP lysis buffer 
(Thermo Fisher Scientific, MA, USA), and centrifuged. The supernatant was then immunoprecipitated with a specific Ab against PEA3 (Santa Cruz, CA, USA) or c-Jun (Santa Cruz, CA, USA) in the presence of protein $A / G$ beads at $4^{\circ} \mathrm{C}$ overnight. The immunoprecipitated beads were washed three times with IP lysis buffer. The immune complex was analyzed through $8 \%$ SDS-PAGE, transferred to PVDF membranes, and then subjected to immunoblot analysis with Abs specific for serine (Abcam, Cambridge, UK), PEA3 (Santa Cruz, CA, USA), or c-Jun (Santa Cruz, CA, USA).

\subsection{Chromatin immunoprecipitation (ChIP)}

WI-38 cells were subjected to hypoxia $\left(1 \% \mathrm{O}_{2}\right)$ for 30 minutes and then fixed with $10 \%$ formaldehyde for 10 minutes. Cells were collected and subjected to sonication; then, anti-PEA3, or anti-c-Jun was used for immunoprecipitation, and mouse anti-IgG antibody was used as the control. The Pref-1 promoter region was amplified through the polymerase chain reaction, and the following primers were used: AP-1, 5'ACCACGAGTCAGCTGGGTAT-3' (sense) and 5'-TGCACACCCAAACACGCAAA-3' (antisense) and PEA3, 5'TTGTGTTTCAGCGCGGCTA-3' (sense) and 5'-CAAGCGGACCTGCGGTTA-3' (antisense). DNA was analyzed with $1 \%$ agarose gel containing ethidium bromide.

\subsection{Study approval}

All animal protocols were approved by the Animal Ethics Committee of Taipei Medical University (approval no. LAC-2016-0361 and LAC-2019-0042).

\subsection{Statistical analysis}

All experimental data are presented as the mean value \pm standard error of the mean for at least three independent experiments. The results of comparisons were conducted using one-way analysis of variance (ANOVA) followed by Dunnett's test analysis, unpaired t test, or Mann-Whitney $U$ test. The results were considered statistically significant if $p$ was $<0.05$.

\section{Results}

\subsection{Colocalization of Pref-1 and HIF-1a in lung sections from OVA-treated mice}

To evaluate Pref- 1 expression in severe asthma, we used a mouse model sensitized with the allergen OVA. Mice received an intraperitoneal injection of $50 \mu \mathrm{g}$ OVA in $2 \mathrm{mg}$ aluminium hydroxide or PBS alone on days 1,7 , and 14 (Fig. 1A). These mice were challenged with aerosolized $5 \%$ OVA twice per week from day 21 to 81 and were then sacrificed for further analysis. In the OVA-sensitized mouse model, colocalization of Pref-1 and HIF-1a was observed through dual-label immunofluorescent staining (Fig. 1B). Moreover, we examined PEA3 expression in the lung section. Increased PEA3 expression was observed in the subepithelial layer of OVA-treated mice through IHC staining (Fig. 1C). These results suggest that Pref-1 and PEA3 are involved in hypoxia-induced airway fibrosis in OVA-sensitized mouse. 


\subsection{Hypoxia-induced Pref-1 mRNA and protein upregulation in WI-38 cells}

Hypoxia induced Pref-1 expression in preadipocytes (34). A study showed that Pref- 1 is involved in human adipose tissue fibrosis (35). However, the mechanism by which hypoxia induces Pref- 1 expression in human lung fibrosis remains unknown. Our data demonstrated that the Pref- 1 mRNA level increased after hypoxic stimulation of WI-38 cells for 1 hour (Fig. 2A). Furthermore, we found that Pref- 1 protein expression was upregulated with a decrease in oxygen concentration (Fig. 2B). These data indicated that hypoxia induced Pref-1 expression in WI-38 cells.

\subsection{PEA3 was involved in hypoxia-induced Pref-1 expression in WI-38 cells}

To determine the role of PEA3 in hypoxia-induced Pref- 1 expression in human lung fibroblasts, WI-38 cells were treated with PEA3 siRNA $(50 \mathrm{ng} / \mathrm{mL})$ overnight and then subjected to hypoxia $\left(1 \% \mathrm{O}_{2}\right)$. We found that hypoxia-induced Pref-1 expression significantly decreased after PEA3 siRNA transfection (Fig. 3A). Moreover, we exposed WI-38 cells to hypoxia for various time intervals. PEA3 serine phosphorylation was observed at 10 minutes, which declined after 60 minutes of exposure (Fig. 3B). We used the NCBI database to predict the transcription factor binding site in the promoter region of Pref- 1 ; we found that PEA3 and AP-1 are the binding sequences in the promoter region of Pref-1. Furthermore, the ChIP assay showed PEA3 binding to the promoter region of Pref-1 in WI-38 cells during hypoxia (Fig. 3C). Hypoxiastimulated cells showed a increase in PEA3-luciferase activity at 24 hours (Fig. 3D). PEA3 was translocated to the nucleus from the cytoplasm after hypoxia in WI-38 cells, as revealed by immunocytochemistry (Fig. 3E). Taken together, these data indicated that PEA3 activation was involved in hypoxia-induced upregulation of Pref-1 expression.

\subsection{Involvement of ERK phosphorylation in PEA3 and AP-1 activation for hypoxia-induced Pref-1 expression in WI-38 cells}

PEA3 plays a role in hypoxia-induced Pref- 1 expression. A study indicated that ERK plays a crucial role in PEA3 activation in gastric adenocarcinoma $(36,37)$. We aimed to elucidate whether hypoxia induced Pref-1 expression through ERK/PEA3/AP-1 signaling in WI-38 cells. Treatment of cells with U0126 (10 $\mu \mathrm{M})$, an ERK inhibitor, downregulated hypoxia-induced Pref-1 expression (Fig. 4A). Moreover, U0126 downregulated hypoxia-induced PEA3 and c-Jun phosphorylation in Wl-38 cells (Fig. 4B, C). These results demonstrated that ERK mediated hypoxia-induced Pref-1 expression, PEA3 and c-Jun phosphorylation in WI-38 cells. 


\subsection{Formation of the AP-1-PEA3 complex mediated Pref-1 gene expression in WI-38 cells}

A study showed that the AP-1-PEA3 complex binds to $I L-8 / C X C L 8$ promoter in human hepatocellular carcinoma (32). Whether the AP-1-PEA3 complex is involved in hypoxia-induced Pref-1 expression remains unknown. In this study, PEA3 antibodies coprecipitated PEA3 and c-Jun after exposure to hypoxia in WI-38 cells (Fig. 5A). Moreover, curcumin (10 $\mu \mathrm{M})$, an AP-1 inhibitor, attenuated hypoxiainduced Pref-1 expression in WI-38 cells (Fig. 5B). We observed that AP-1 bound to the promoter of Pref-1 after hypoxia exposure in the ChIP assay (Fig. 5C). Collectively, the results showed that AP-1-PEA3 complex was involved in Pref-1 expression in WI-38 cells.

\section{Discussion}

The present study demonstrated that hypoxia induces ERK phosphorylation, which in turn activates PEA3 and AP-1 that binds to the promoter region of Pref-1; this upregulates Pref-1 expression in human lung fibroblast cells. These results indicate that hypoxia-induced Pref-1 may play a crucial role in airway fibrosis.

In fibroblastic lung diseases, fibroblast proliferation and differentiation can rapidly decline lung function $(38,39)$. Studies have demonstrated that moderate to severe hypoxia $(0.1-5 \%)$ can promote the proliferation of airway smooth muscle cells and lung fibroblasts $(1,39,40)$. However, the correlation of hypoxia and Pref-1 with airway fibrosis remains unknown. Moreover, HIF-1 a regulates hypoxia-induced lung fibroblast proliferation (39). In the present study, Pref-1 and HIF-1 a colocalized in the OVA-induced allergic airway. It can be implied that Pref- 1 is associated with hypoxic airway fibrosis. However, the mechanism of Pref-1-induced lung fibroblast differentiation requires further investigation.

Pref-1 suppressed adipogenesis by binding to the integrin receptor and then inhibited C/EBP $\beta$ and C/EBPS genes in preadipocytes (22). Pref-1 showed strong correlations with various metabolic conditions, including hepatic steatosis, blood pressure, and insulin sensitivity (41). Moreover, Pref-1 impaired proinflammatory cytokine expression in human bone marrow mesenchymal stem cells (42). These findings suggest that Pref- 1 is involved in chronic inflammation and metabolic diseases. A study reported that hypoxia induces Pref- 1 expression in preadipocytes during adipogenesis but does not increase Pref- 1 levels in adipocytes because hypoxia alters histone modification in preadipocytes (34). In this study, hypoxia upregulated Pref-1 mRNA and protein expression through the ERK/PEA3/AP-1 cascade in human lung fibroblasts.

A study showed that PEA3 is involved in many processes, including epithelial mesenchymal transition, apoptosis, cell invasion, and chemotherapy resistance (43). A study showed that mitogen-activated protein kinase (MAPK) signaling upregulated PEA3 expression (36). Moreover, PI3K/Akt signaling activated PEA3 expression in renal cell carcinoma (44). In this study, PEA3 was activated with ERK, and then, it bound to the Pref-1 promoter region after hypoxia exposure in WI-38 cells. We found that hypoxia 
induced PEA3 phosphorylation and translocation to the nucleus, which in turn induced Pref- 1 expression in WI-38 cells. Furthermore, PEA3 siRNA downregulated hypoxia-induced Pref- 1 expression. These results demonstrated that PEA3 contributed to hypoxia-induced Pref-1 expression.

MAPK/ERK regulates lung fibrogenesis and cell growth and proliferation (45). Several studies have reported that ERK is essential for mediating profibrotic gene expression through the activation of transcriptional factors, including AP-1 $(33,46)$. MAPK/ERK regulates transforming growth factor $\beta 1$ induced human alveolar type II cell senescence and epithelial mesenchymal transition (47). Moreover, hypoxia induces ERK Tyr204 phosphorylation, which in turn contributes to AP-1 activation and connective tissue growth factor expression in WI-38 cells (48). In the present study, ERK was involved in hypoxiainduced PEA3 phosphorylation, c-Jun phosphorylation and Pref-1 expression in human lung fibroblasts. Thus, hypoxia induced Pref-1 expression through the ERK/PEA3/AP-1 pathway.

A study demonstrated that hypoxia induced AP-1 expression and promoted downstream gene expression (30). A study showed that hypoxia induced AP-1 phosphorylation and contributed to fibrogenic protein expression in human lung fibroblasts (48). In retinal vascular endothelial cells, AP-1 activated JNK under hypoxia and then induced Cyr61 protein expression (30). Here, we demonstrated that curcumin inhibited hypoxia-induced Pref-1 expression, and the AP-1-PEA3 complex was recruited to the Pref-1 promoter after hypoxia in human lung fibroblasts.

In conclusion, these findings showed that hypoxia activates the ERK/PEA3/AP-1 signaling pathway, which in turn. Figure 6 shows a simplified diagram of the signaling pathway that demonstrates hypoxiainduced Pref-1 upregulation in human lung fibroblasts. Our findings demonstrate how hypoxia induces Pref-1 expression in human lung fibroblasts.

\section{Declarations}

\section{Ethics approval and consent to participate}

All animal protocols were approved by the Animal Ethics Committee of Taipei Medical University (approval no. LAC-2016-0361 and LAC-2019-0042).

\section{Availability of data and materials}

Not applicable.

\section{Conflict of interest}

The authors declare no conflict of interest.

\section{Funding}

MOST108-2320-B-038-0068 and MOST109-2320-B-038-068 from the Ministry of Science and Technology of Taiwan, R.O.C. 


\section{Author Contributions}

Conceptualization: WHC, BCC.

Data curation: WHC, BCC.

Formal analysis: WHC, BCC.

Funding acquisition: WHC, BCC. Investigation: WHC, BCC.

Methodology: CLC, JYC, BCC. Project administration: CLC BCC.

Resources: BCC. Software: WHC, BCC.

Supervision: BCC.

Validation: WHC, CLC, CHL, BCC. Visualization: WHC, CLC, CHL, BCC

Writing - original draft: WHC. Writing - review \& editing: BCC.

\section{Acknowledgment}

This study was supported by grants (MOST109-2320-B-038-068) from the Ministry of Science and Technology of Taiwan, R.O.C.

\section{References}

1. Ahmad T, et al. (2012) Hypoxia response in asthma: differential modulation on inflammation and epithelial injury. Am J Respir Cell Mol Biol47: 1-10.

2. Hough KP, et al. (2020) Airway Remodeling in Asthma. Front Med (Lausanne)7: 191.

3. Hekking PP, et al. (2015) The prevalence of severe refractory asthma. J Allergy Clin Immuno/135: 896-902.

4. Thomson CC, et al. (2014) Severe asthma. Ann Am Thorac Soc11: 996-997.

5. Bergeron C, Tulic MK, Hamid Q. (2010) Airway remodelling in asthma: from benchside to clinical practice. Can Respir J17: e85-93.

6. Hoshino M, Nakamura Y, Sim J, Shimojo J, Isogai S. (1998) Bronchial subepithelial fibrosis and expression of matrix metalloproteinase-9 in asthmatic airway inflammation. J Allergy Clin Immuno/102: 783-788.

7. Brewster CE, et al. (1990) Myofibroblasts and subepithelial fibrosis in bronchial asthma. Am J Respir Cell Mol Bio/3: 507-511.

8. Chetta A, et al. (1997) Airways remodeling is a distinctive feature of asthma and is related to severity of disease. Chest111: 852-857. 
9. Little SA, et al. (2002) High resolution computed tomographic assessment of airway wall thickness in chronic asthma: reproducibility and relationship with lung function and severity. Thorax57: 247253.

10. Tanaka T. (2016) Expanding roles of the hypoxia-response network in chronic kidney disease. Clin Exp Nephro/20: 835-844.

11. Rosmorduc O, Housset C. (2010) Hypoxia: a link between fibrogenesis, angiogenesis, and carcinogenesis in liver disease. Semin Liver Dis30: 258-270.

12. Chai $X$, et al. (2018) Hypoxia induces pulmonary arterial fibroblast proliferation, migration, differentiation and vascular remodeling via the PI3K/Akt/p70S6K signaling pathway. Int J Mol Med41: 2461-2472.

13. Senavirathna LK, et al. (2018) Hypoxia induces pulmonary fibroblast proliferation through NFAT signaling. Sci Rep8: 2709.

14. Pugh CW, O'Rourke JF, Nagao M, Gleadle JM, Ratcliffe PJ. (1997) Activation of hypoxia-inducible factor-1; definition of regulatory domains within the alpha subunit. J Biol Chem272: 11205-11214.

15. Polosukhin VV, et al. (2007) Association of progressive structural changes in the bronchial epithelium with subepithelial fibrous remodeling: a potential role for hypoxia. Virchows Arch451: 793803.

16. Kostakou E, et al. (2019) Acute Severe Asthma in Adolescent and Adult Patients: Current Perspectives on Assessment and Management. J Clin Med8.

17. Kang HH, et al. (2020) The Effects of Chronic Intermittent Hypoxia in Bleomycin-Induced Lung Injury on Pulmonary Fibrosis via Regulating the NF-kappaB/Nrf2 Signaling Pathway. Tuberc Respir Dis (Seoul)83: S63-S74.

18. Braun RK, et al. (2018) Chronic intermittent hypoxia worsens bleomycin-induced lung fibrosis in rats. Respir Physiol Neurobio/256: 97-108.

19. Guo L, Xu JM, Liu L, Liu SM, Zhu R. (2015) Hypoxia-Induced Epithelial-Mesenchymal Transition Is Involved in Bleomycin-Induced Lung Fibrosis. Biomed Res Int2015: 232791.

20. Wang Y, Zhao L, Smas C, Sul HS. (2010) Pref-1 interacts with fibronectin to inhibit adipocyte differentiation. Mol Cell Bio/30: 3480-3492.

21. Wang Y, Kim KA, Kim JH, Sul HS. (2006) Pref-1, a preadipocyte secreted factor that inhibits adipogenesis. J Nutr136: 2953-2956.

22. Wang Y, Sul HSJCm. (2009) Pref-1 regulates mesenchymal cell commitment and differentiation through Sox9 9: 287-302.

23. Zhu NL, et al. (2012) Hepatic stellate cell-derived delta-like homolog 1 (DLK1) protein in liver regeneration. J Biol Chem287: 10355-10367.

24. Tschope C, Diez J. (2019) Myocardial fibrosis as a matter of cell differentiation: opportunities for new antifibrotic strategies. Eur Heart J40: 979-981. 
25. Chen JY, Lin CH, Chen BC. (2017) Hypoxia-induced ADAM 17 expression is mediated by RSK1dependent C/EBPbeta activation in human lung fibroblasts. Mol Immuno/88: 155-163.

26. de Launoit $Y$, et al. (2006) The Ets transcription factors of the PEA3 group: transcriptional regulators in metastasis. Biochim Biophys Acta1766: 79-87.

27. Warburton D, Shi W, Xu B. (2013) TGF-beta-Smad3 signaling in emphysema and pulmonary fibrosis: an epigenetic aberration of normal development? Am J Physiol Lung Cell Mol Physio/304: L83-85.

28. O'Hagan RC, Tozer RG, Symons M, McCormick F, Hassell JA. (1996) The activity of the Ets transcription factor PEA3 is regulated by two distinct MAPK cascades. Oncogene13: 1323-1333.

29. Ratovitski EA. (2010) LKB1/PEA3/DeltaNp63 pathway regulates PTGS-2 (COX-2) transcription in lung cancer cells upon cigarette smoke exposure. Oxid Med Cell Longev3: 317-324.

30. You J-J, Yang C-M, Chen M-S, Yang C-HJEer. (2010) Regulation of Cyr61/CCN1 expression by hypoxia through cooperation of C-Jun/AP-1 and HIF-1a in retinal vascular endothelial cells 91: 825836.

31. Weng T, et al. (2014) Hypoxia-induced deoxycytidine kinase contributes to epithelial proliferation in pulmonary fibrosis. American journal of respiratory critical care medicine190: 1402-1412.

32. Iguchi A, et al. (2000) PEA3 and AP-1 are required for constitutive IL-8 gene expression in hepatoma cells. Biochem Biophys Res Commun279: 166-171.

33. Lin C-H, et al. (2014) CXCL12 Induces Connective Tissue Growth Factor Expression in Human Lung Fibroblasts through the Rac1/ERK, JNK, and AP-1 Pathways. PLOS ONE9: e104746.

34. Moon Y, Lee S, Park B, Park H. (2018) Distinct hypoxic regulation of preadipocyte factor-1 (Pref-1) in preadipocytes and mature adipocytes. Biochim Biophys Acta Mol Cell Res1865: 334-342.

35. Divoux A, et al. (2010) Fibrosis in human adipose tissue: composition, distribution, and link with lipid metabolism and fat mass loss. Diabetes59: 2817-2825.

36. Keld R, et al. (2011) PEA3/ETV4-related transcription factors coupled with active ERK signalling are associated with poor prognosis in gastric adenocarcinoma. Br J Cancer105: 124-130.

37. Guo B, Sharrocks AD. (2009) Extracellular signal-regulated kinase mitogen-activated protein kinase signaling initiates a dynamic interplay between sumoylation and ubiquitination to regulate the activity of the transcriptional activator PEA3. Mol Cell Bio/29: 3204-3218.

38. King TE, Jr., Pardo A, Selman M. (2011) Idiopathic pulmonary fibrosis. Lancet378: 1949-1961.

39. Mizuno S, et al. (2009) Hypoxia regulates human lung fibroblast proliferation via p53-dependent and -independent pathways. Respir Res10: 17.

40. Cogo A, et al. (2003) Effects of hypoxia on rat airway smooth muscle cell proliferation. J App/ Physiol (1985)94: 1403-1409.

41. O'Connell J, Lynch L, Hogan A, Cawood TJ, O'Shea D. (2011) Preadipocyte factor-1 is associated with metabolic profile in severe obesity. J Clin Endocrinol Metab96: E680-684.

42. Abdallah BM, et al. (2007) dlk1/FA1 regulates the function of human bone marrow mesenchymal stem cells by modulating gene expression of pro-inflammatory cytokines and immune response- 
related factors. J Biol Chem282: 7339-7351.

43. Qi T, et al. (2020) Function and regulation of the PEA3 subfamily of ETS transcription factors in cancer. Am J Cancer Res10: 3083-3105.

44. Xu L, et al. (2020) ETV4 is a theranostic target in clear cell renal cell carcinoma that promotes metastasis by activating the pro-metastatic gene FOSL1 in a PI3K-AKT dependent manner. Cancer Lett482: 74-89.

45. Madala SK, et al. (2012) MEK-ERK pathway modulation ameliorates pulmonary fibrosis associated with epidermal growth factor receptor activation. Am J Respir Cell Mol Bio/46: 380-388.

46. Engers R, et al. (2006) Rac upregulates tissue inhibitor of metalloproteinase-1 expression by redoxdependent activation of extracellular signal-regulated kinase signaling. FEBS J273: 4754-4769.

47. Chen $\mathrm{H}$, et al. (2020) TGF-beta1/IL-11/MEK/ERK signaling mediates senescence-associated pulmonary fibrosis in a stress-induced premature senescence model of Bmi-1 deficiency. Exp Mol Med52: 130-151.

48. Cheng Y, et al. (2017) Induction of Connective Tissue Growth Factor Expression by Hypoxia in Human Lung Fibroblasts via the MEKK1/MEK1/ERK1/GLI-1/GLI-2 and AP-1 Pathways. PLoS One12: e0188608.

\section{Figures}


A

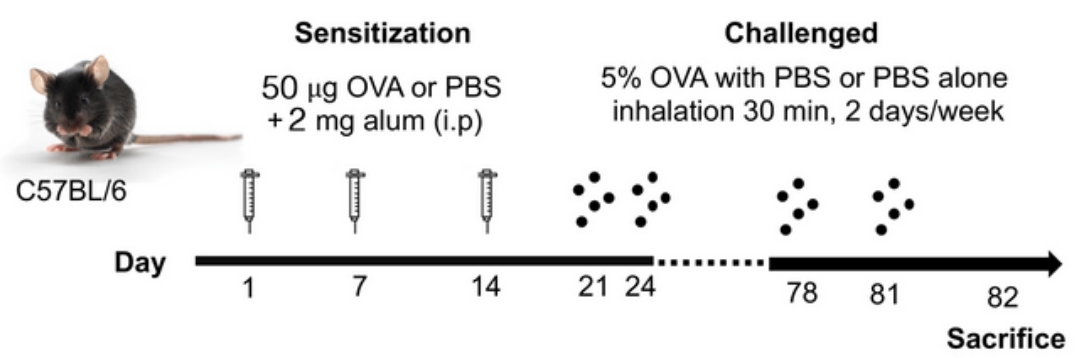

B
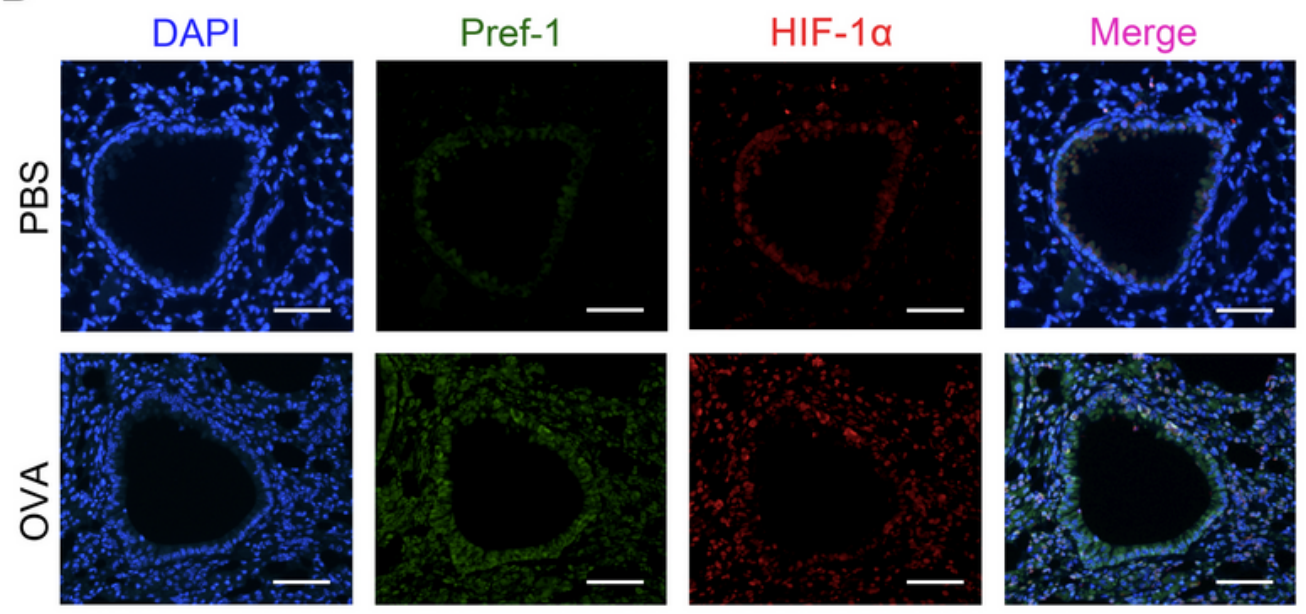

C
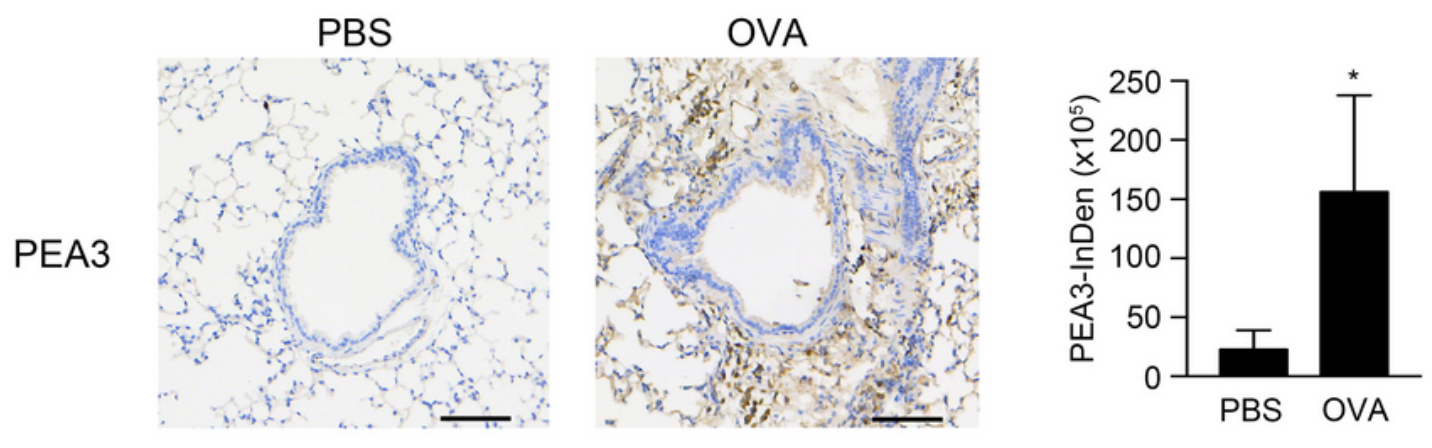

\section{Figure 1}

Pref-1 and PEA3 expression in lung tissue sections obtained from OVA-treated mice. (A) Mice were sensitized with OVA through three intraperitoneal injections on days 1,7 , and 14 with OVA absorbed to aluminium hydroxide. Mice were sensitized and challenged with aerosolized OVA twice weekly for up to 82 days. Control mice were treated with PBS. Lung tissue sections were stained through (B) immunofluorescence for Pref-1 (green) and HIF-1a (red) and (C) immunohistochemistry for PEA3 
expression (original magnification, 20x; $n=5$ ). PEA3 Integrated density are presented as the mean \pm SEM. ${ }^{*} \mathrm{p}<0.05$, compared with PBS.

A

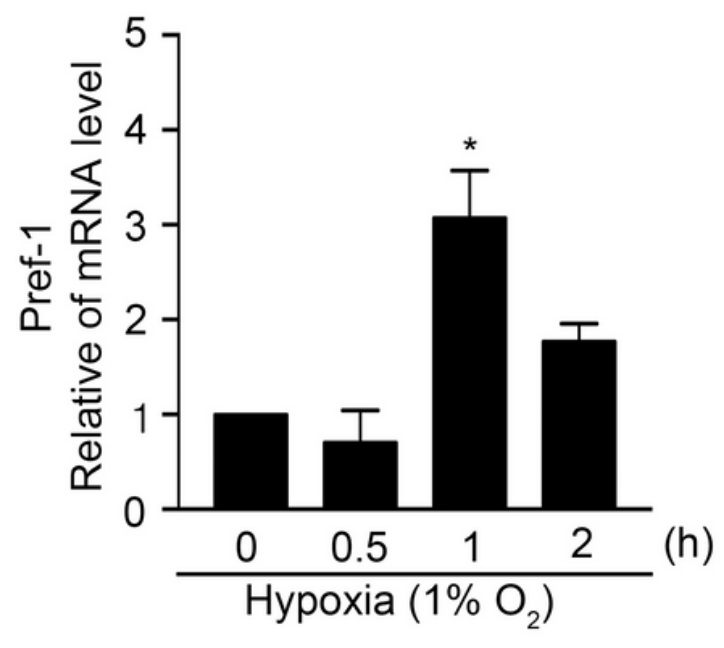

B

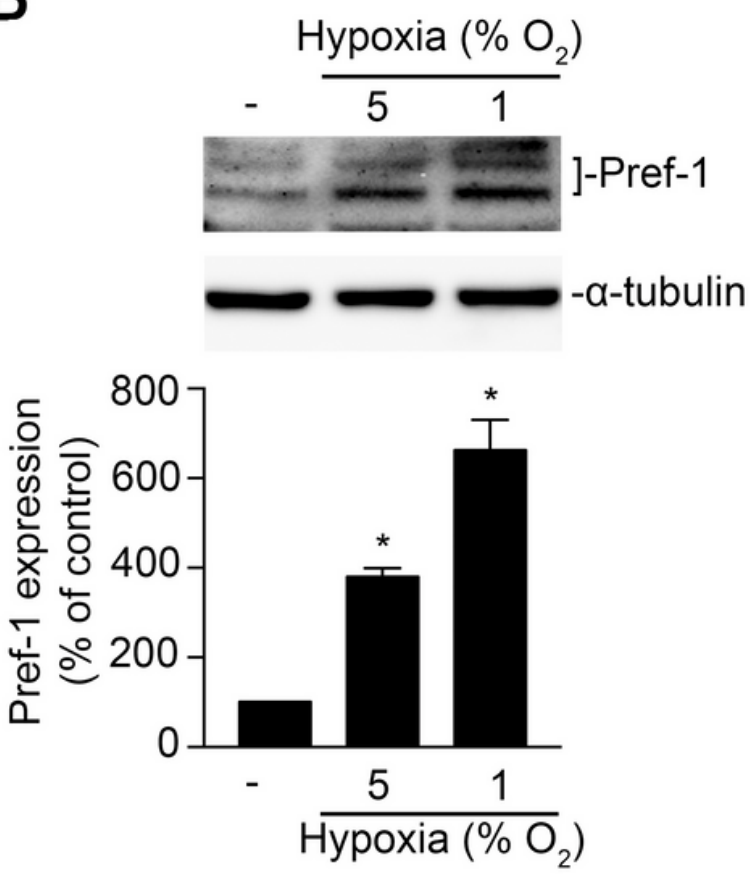

Figure 2

Hypoxia-induced Pref- 1 expression in WI-38 cells. (A) WI-38 cells were incubated with $1 \% 02$ for $0-2$ hours. The levels of Pref- 1 messenger RNA were detected by using qPCR . Data are presented as the mean \pm SEM of three experiments. (B) WI-38 cells were incubated with hypoxia for different concentrations, and then, Pref- 1 and a-tubulin were determined through immunoblots. These are presented as the mean $\pm \operatorname{SEM}(n=3)$. ${ }^{\star} p<0.05$, compared with control (at $\left.0221 \%\right)$. 
A

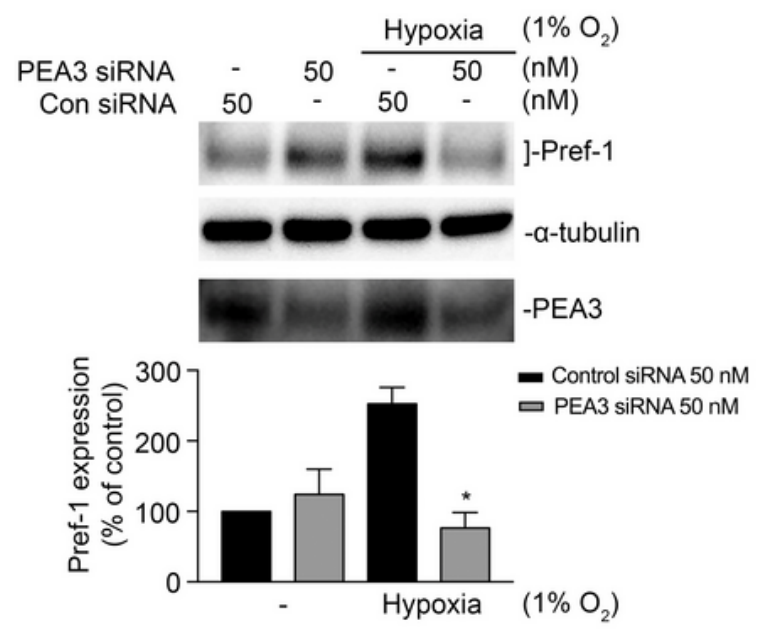

C

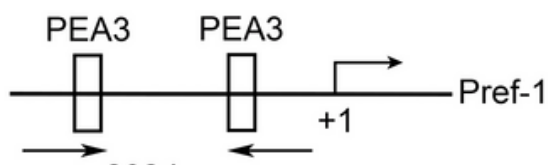

202 b.p.

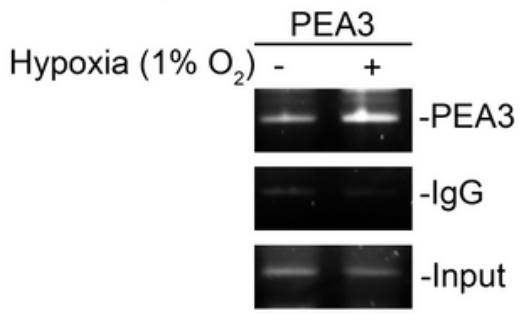

$E$
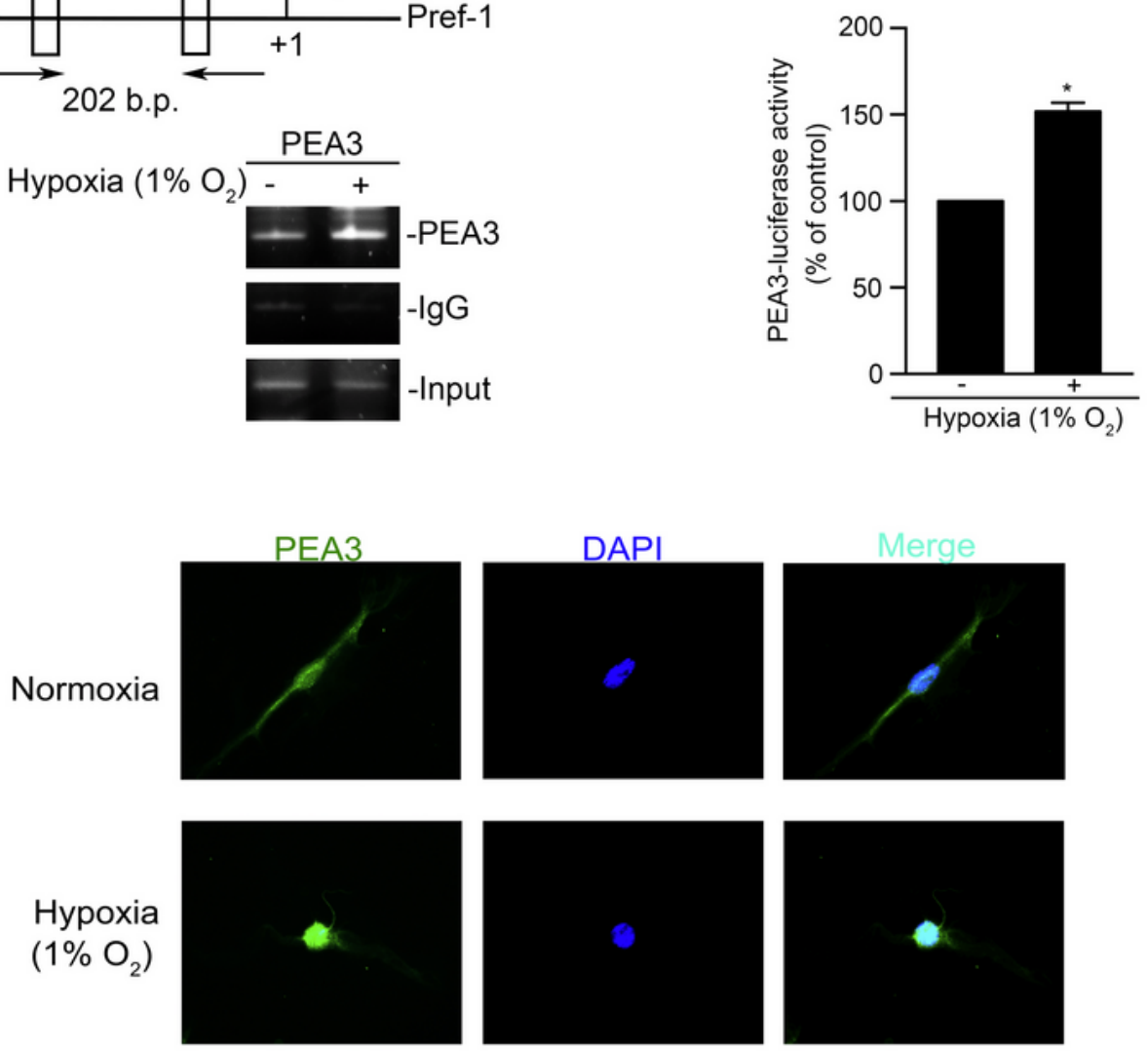

\section{Figure 3}

Involvement of PEA3 in hypoxia-induced Pref-1 expression in WI-38 cells. (A) WI-38 cells were transfected with control siRNA or PEA3 siRNA for 24 hours and then subjected to hypoxia (1\% 02) for another 4 hours. Western blotting was performed to assess the levels of Pref-1, a-tubulin, and PEA3 in cell lysates. Data are presented as the mean \pm SEM of three experiments. ${ }^{*} p<0.05$, compared with the control siRNA group. (B) WI-38 cells were subjected to hypoxia for the indicated time, and cell lysates were 
immunoprecipitated with an anti-PEA3 antibody; further, they were immunoblotted with antibodies for serine and PEA3. Data are presented as the mean \pm SEM of three experiments. ${ }^{*} p<0.05$, compared with the control at 0 minutes. (C) Cells were subjected to hypoxia (1\% 02) for 30 minutes; the PEA3 binding site of the Pref-1 promoter region was detected through the ChIP assay. Input for for use as a positive control. Mouse polyclonal IgG for use as a negative control. Traces indicate that the three experiments produced similar results. (D) Cells were transfected with $0.8 \mu \mathrm{g}$ of PEA3-Luc and $0.1 \mu \mathrm{g}$ of pBK-CMV-Lac $Z$ for 24 hours and then subjected to hypoxia ( $1 \% 02)$ for another 16 hours. Cells were harvested for a luciferase activity assay. Data are shown as the mean $\pm S E M, n=3 .{ }^{*} p<0.05$, relative to nonstimulated cells. (E) WI-38 cells were subjected to hypoxia (1\% 02) for 30 minutes. In confocal microscopy, the cells were incubated with antibodies specific for PEA3, and immunoreactivity was performed through the incubation of the cells with an FITC-conjugated secondary antibody. All slides were counterstained with DAPI (blue) to distinguish the nucleus, which were visualized under an immunofluorescence microscopy (original magnification, 20x; $\mathrm{n}=3$ ). 
A

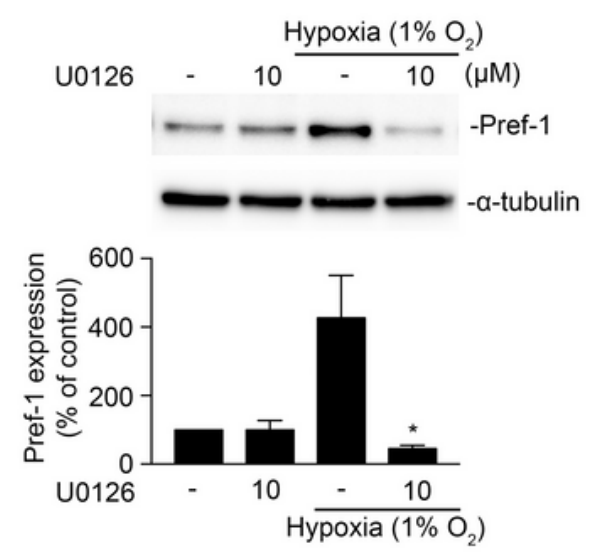

B
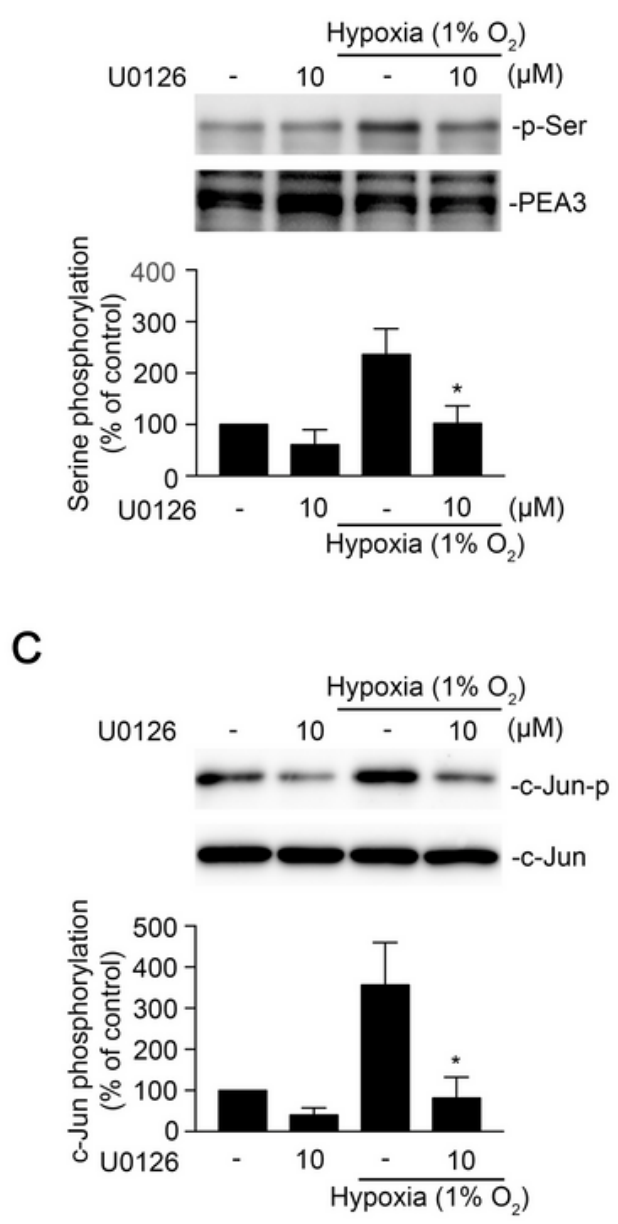

\section{Figure 4}

Involvement of ERK in hypoxia induces a Pref- 1 expression in WI-38 cells. (A) WI-38 cells were pretreated with $\mathrm{U} 0126$ for 30 minutes and then incubated with the hypoxia (1\% 02) for 4 hours. Levels of Pref- 1 and a-tubulin in cell lysates were determined. Data are presented as the mean \pm SEM of three experiments. *p $<0.05$, compared with the hypoxia-exposed group. Cells were pretreated with U0126 for 30 minutes and then subjected to hypoxia (1\% 02) for 30 minutes. (B) PEA3 was immunoprecipitated using anti-PEA3 
antibody, and PEA3-p-serine was detected using anti-p-serine antibody. The quantified results were adjusted with PEA3 and expressed as a percentage of control. (C) Levels of phosphor-c-Jun Ser63 and cJun were detected using Western blotting in cell lysates. Values represent the means \pm SEM of three experiments. ${ }^{*} p<0.05$.

A

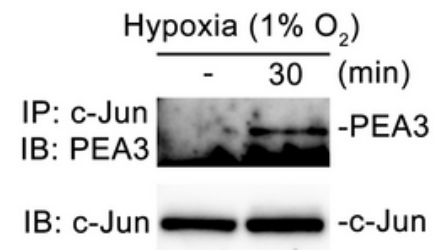

B
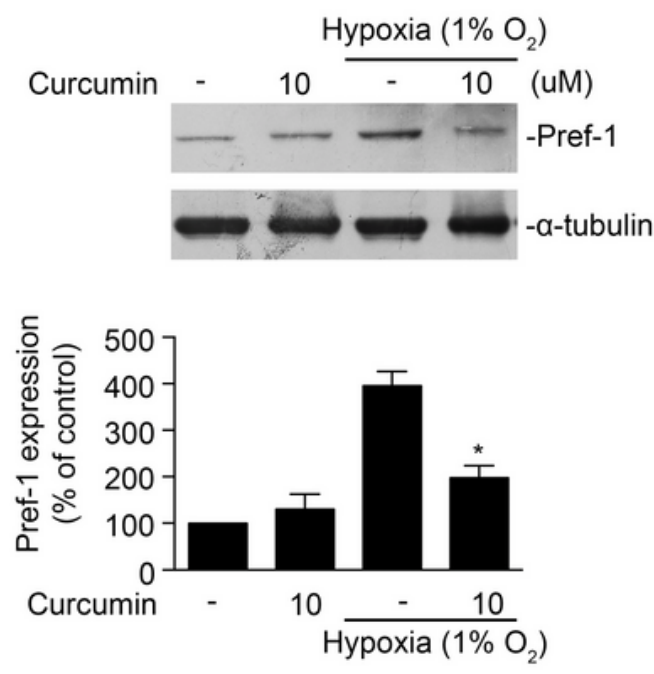

C

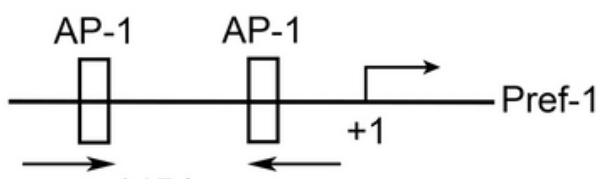

145 b.p.

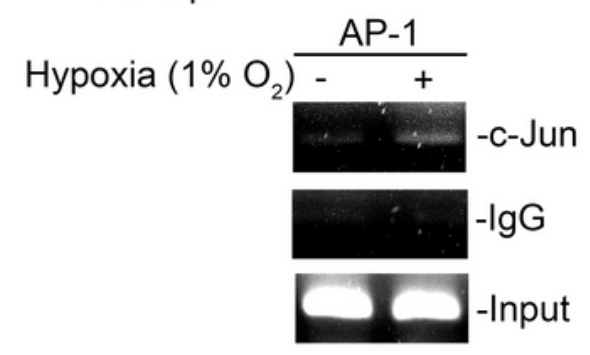

Figure 5 
Involvement of AP-1 in hypoxia-induced Pref-1 expression in WI-38 cells. (A) WI-38 cells were exposed to hypoxia ( $1 \% 02)$ for 30 minutes. Cells were lysed with IP lysis buffer and then immunoprecipitated with the anti-c-Jun antibody. The immunoprecipitated complex was detected through immunoblotting with an anti-PEA3 antibody. Typical traces were demonstrative of three experiments. (B) Cells were pretreated with curcumin $(10 \mu \mathrm{M})$ for 30 minutes and then stimulated with hypoxia $(1 \% 02)$ for another 4 hours. Pref- 1 and a-tubulin were detected in cell lysates. Data are presented as the mean \pm SEM for three independent experiments. ${ }^{*} \mathrm{p}<0.05$, compared with the hypoxia group. (C) WI-38 cells were exposed to hypoxia (1\% 02) for 30 minutes. The AP-1 binding site of the Pref-1 promoter region was detected through ChIP assay. Typical traces were presented in all two experiments.

\section{Hypoxia}

\section{Human Lung Fibroblasts}

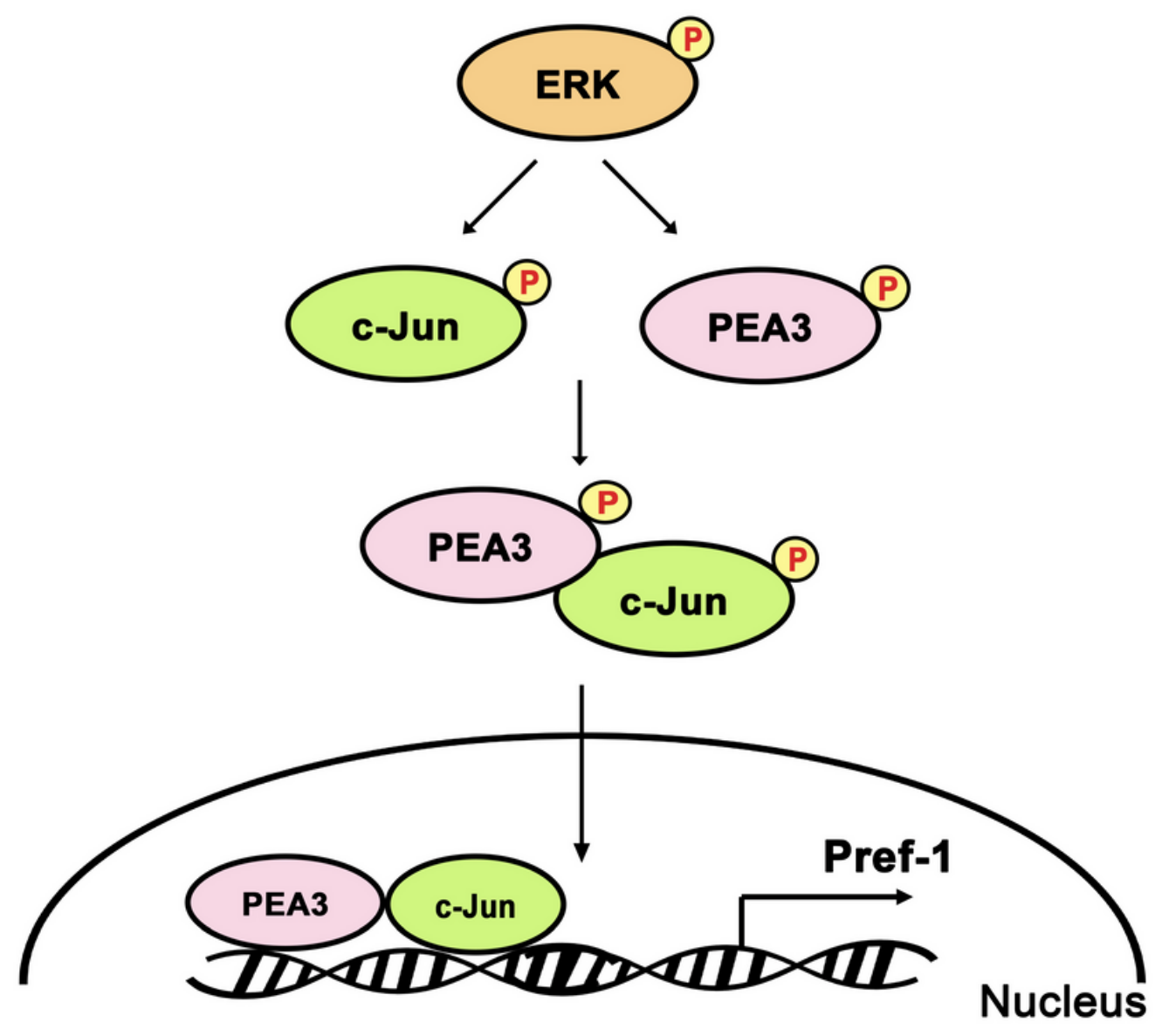

Figure 6 
Simplified image displaying the results of the expression of hypoxia-induced Pref- 1 through the ERK/PEA3/AP-1 pathway in human lung fibroblasts. Hypoxia induced the activation of ERK, which in turn caused PEA3 and AP-1 phosphorylation and complex formation. Moreover, PEA3 and AP-1 complex mediates Pref-1 expression through hypoxia stimulation in human lung fibroblasts. 